OPEN ACCESS

Edited by:

Yanjiao Zhou,

UConn Health, United States

Reviewed by: Vania Aparecida Vicente Aparecida Vicente,

Federal University of Paraná, Brazi

Suresh Chand Bokoliya,

UConn Health, United States

*Correspondence:

Xianghong Liu

Ixh7176@126.com

these authors have contributed equally to this work and share first authorship

Specialty section:

This article was submitted to Infectious Diseases-Surveillance,

Prevention and Treatment, a section of the journa

Frontiers in Medicine

Received: 28 October 2021

Accepted: 10 January 2022

Published: 16 February 2022

Citation:

Yang J, Xie S, Li J, Xia H and Liu X (2022) Brain Abscess Caused by Nocardia farcinica and Diagnosed by

Metagenomic Next-Generation

Sequencing: A Case Report.

Front. Med. 9:803554.

doi: 10.3389/fmed.2022.803554

\section{Brain Abscess Caused by Nocardia farcinica and Diagnosed by Metagenomic Next-Generation Sequencing: A Case Report}

\author{
Jianhua Yang ${ }^{1+}$, Shuhua Xie ${ }^{1+}$, Junda $\mathrm{Li}^{1}, \mathrm{Han}_{\mathrm{Xia}}{ }^{2}$ and Xianghong Liu ${ }^{1 *}$ \\ ${ }^{1}$ Department of Neurology, The Affiliated Ganzhou Hospital of Nanchang University, Ganzhou, China, ${ }^{2}$ Department of \\ Scientific Affairs, Hugobiotech Co., Ltd., Beijing, China
}

Background: Brain abscesses caused by Nocardia farcinica are rare and difficult to diagnose. Conventional methods for diagnosing Nocardia species include blood culture, microscopy, and tissue slice, but the performance is not satisfied. We report a case of brain abscess due to $N$. farcinica diagnosed by metagenomic next-generation sequencing (mNGS).

Case Presentation: We report a case of a 58-year-old man with brain abscess caused by $N$. farcinica. The patient had a history of pemphigus and required long-term methylprednisolone administration. No pathogen was detected in blood culture, cerebrospinal fluid (CSF) culture, and fast-acid staining. mNGS identified $N$. farcinica in the CSF. The symptoms and signs of the patient were significantly improved after changing the antibiotics accordingly to sensitive antibiotics.

Conclusion: Metagenomic next-generation sequencing (mNGS) is helpful for early diagnosis and subsequent treatment of Nocardia-associated meningitis and encephalitis, avoiding brain surgery. Early and accurate diagnosis and prompt antibiotic treatment reduced its mortality.

Keywords: Nocardia farcinica, central nervous system, infection, brain abscess, metagenomic next-generation sequencing

\section{BACKGROUND}

Nocardia is a Gram-positive, branching aerobic bacterium and universal in soil, water, and decaying plant matters. The Nocardia bacterium is an opportunistic pathogen that infects through the respiratory tract or skin contact. Predisposing factors are immunosuppressive treatment (28\%), premedication (26\%), hematological neoplasm (13\%), transplant recipients (13\%), HIV infection (11\%), chronic lung disease (9\%), diabetes mellitus (8\%), and renal disease (8\%) (1). Clinical forms of Nocardia farcinica infection include pulmonary or pleural infections, brain abscesses and skin or soft tissue infections (2). Brain abscesses caused by $N$. farcinica are rare and account for $2 \%$ of all intracranial abscesses (3). However, the mortality rate of nocardial brain abscesses is as high as $30 \%$, which is much higher than the $10 \%$ caused by other bacteria (4), even with the development of imaging technologies and new antibiotics.

The diagnosis of $N$. farcinica using conventional methods is difficult, and the misdiagnosis may lead to inappropriate treatments and delay the therapy, resulting in the high mortality of the disease. Routine diagnostic methods are blood, 
cerebrospinal fluid, and brain aspirate culture, and craniotomy is generally performed to remove a lesion and obtain a specimen. According to the literature, $45.8 \%$ of patients underwent both medical and surgical management (5). Currently, metagenomic next-generation sequencing ( $\mathrm{mNGS}$ ) is being performed to identify pathogens in many infectious diseases, with high sensitivity and specificity (6). The introduction of this technique would contribute to early and accurate diagnosis of the disease, avoid surgery, and reduce mortality. Here, we report a case of $N$. farcinica brain abscess diagnosed by mNGS.

\section{CASE PRESENTATION}

A 58-year-old male with slurred speech for 11 days, accompanied by headache, fever, dizziness and vomiting for 1 day, was admitted to the Department of Neurology at the Affiliated
Ganzhou Hospital of Nanchang University on November 15, 2018. He had a history of tuberculosis in 1978, pemphigus treated with oral methylprednisolone $8 \mathrm{mg}$ per day for 1 year, and fungal pneumonia treated with voriconazole $200 \mathrm{mg}$ twice daily. On examination, his body temperature was $37.1^{\circ} \mathrm{C}$. Neurologic examination revealed nuchal rigidity, dysarthria, and impaired coordination (finger to nose test) of the right upper limb.

Laboratory findings included an initial leukocyte count of 9.85 $\times 10^{9} / \mathrm{L}$ (with $88.1 \%$ polymorphonuclear cells, $5.7 \%$ monocytes, and $6.1 \%$ lymphocytes), ESR of $10 \mathrm{~mm}$ (normal range $0-20 \mathrm{~mm}$ per hour), C-reactive protein of $51 \mathrm{mg} / \mathrm{L}$ (normal range < $10 \mathrm{mg} / \mathrm{L}$ ), and albumin level of $41.3 \mathrm{~g} / \mathrm{L}$. Brain MRI showed cerebellar vermis and right cerebellar mass with peripheral contrast-enhancement and surrounding edema (Figure 1). Chest CT scan revealed chronic interstitial inflammation and localized emphysema of the left upper lung (Figure 2A).

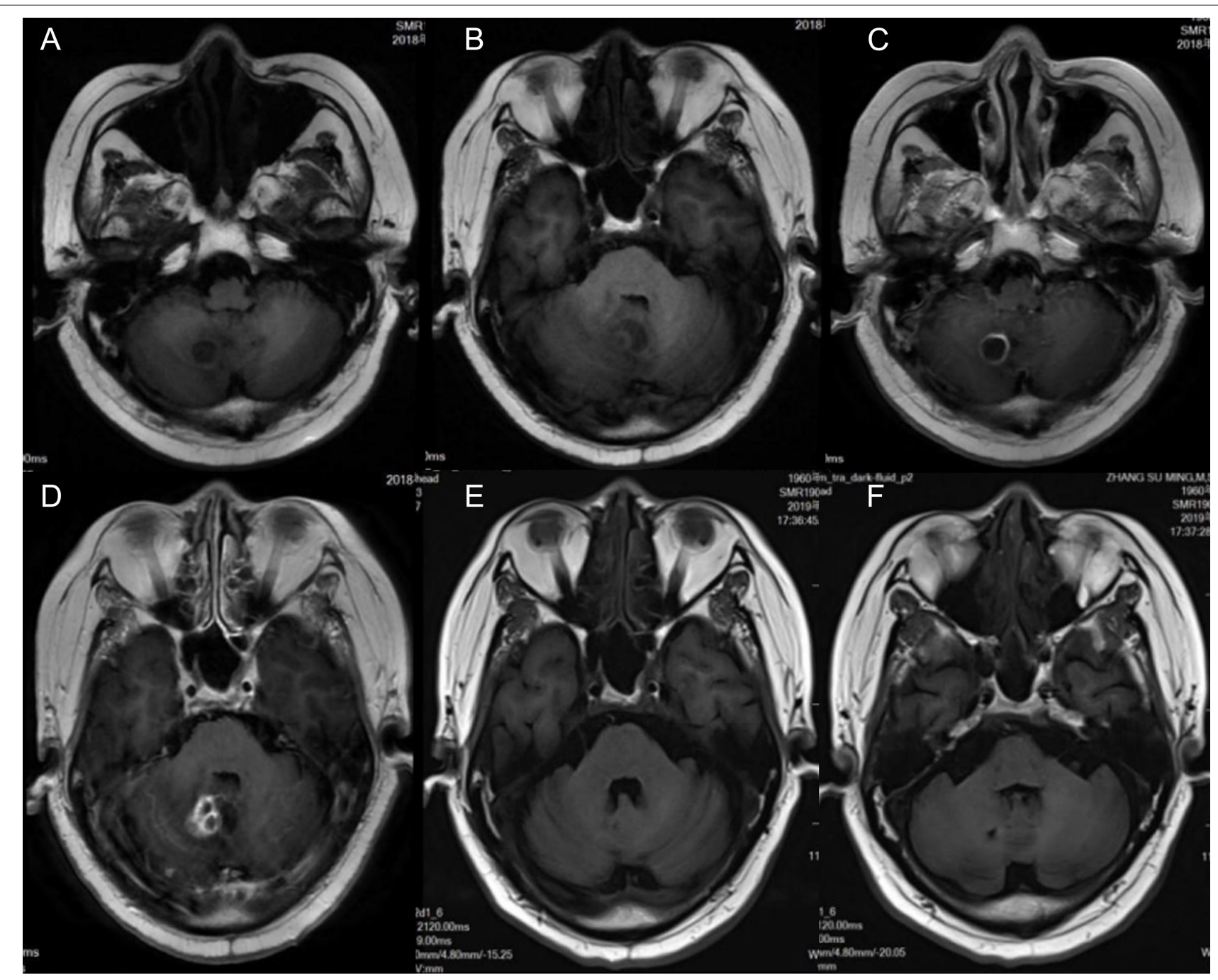

FIGURE 1 | Magnetic resonance imaging (MRI) of the patient shows cerebellar vermis and right cerebellar brain abscess with ring contrast enhancement and peripheral edema. (A,B) T1-weighted imaging. (C,D) Axial and sagittal T1 contrast enhancement imaging. $(\mathbf{E}, \mathbf{F})$ T1-weighted imaging 4 months after treatment; the lesion was reduced markedly. 


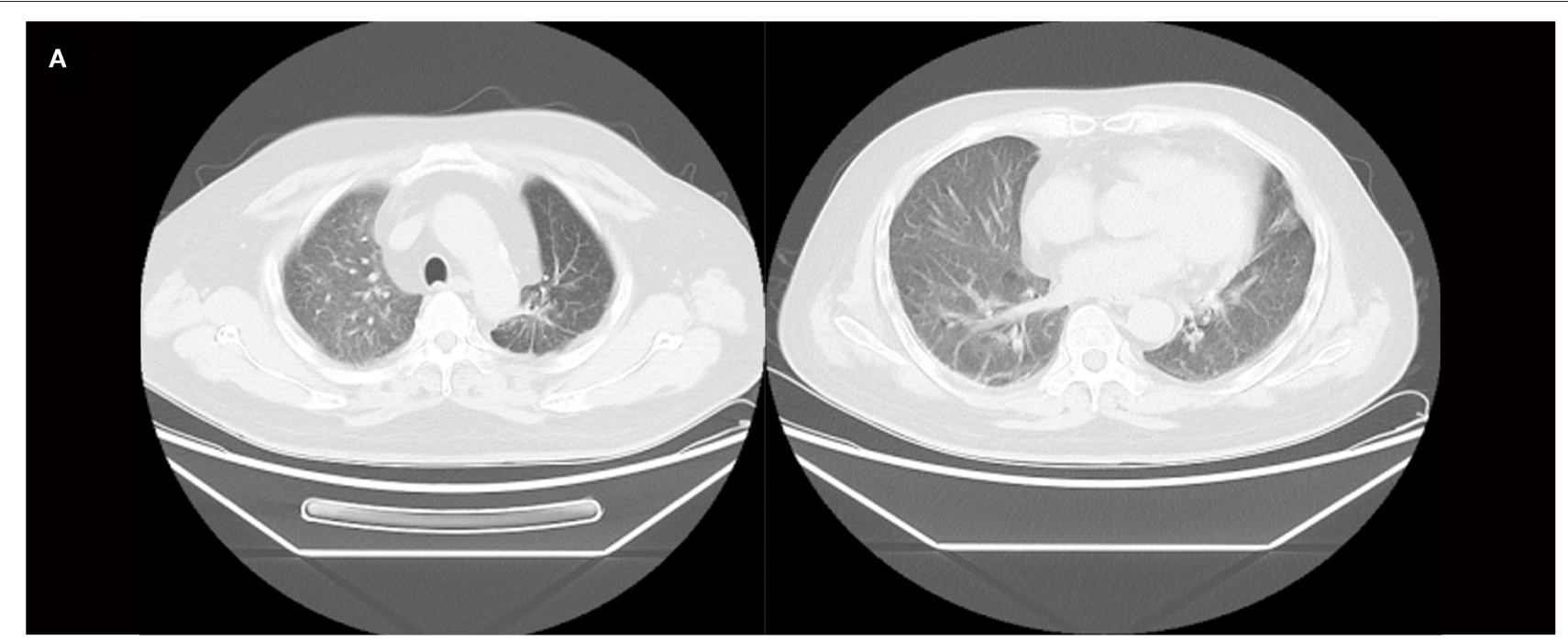

B

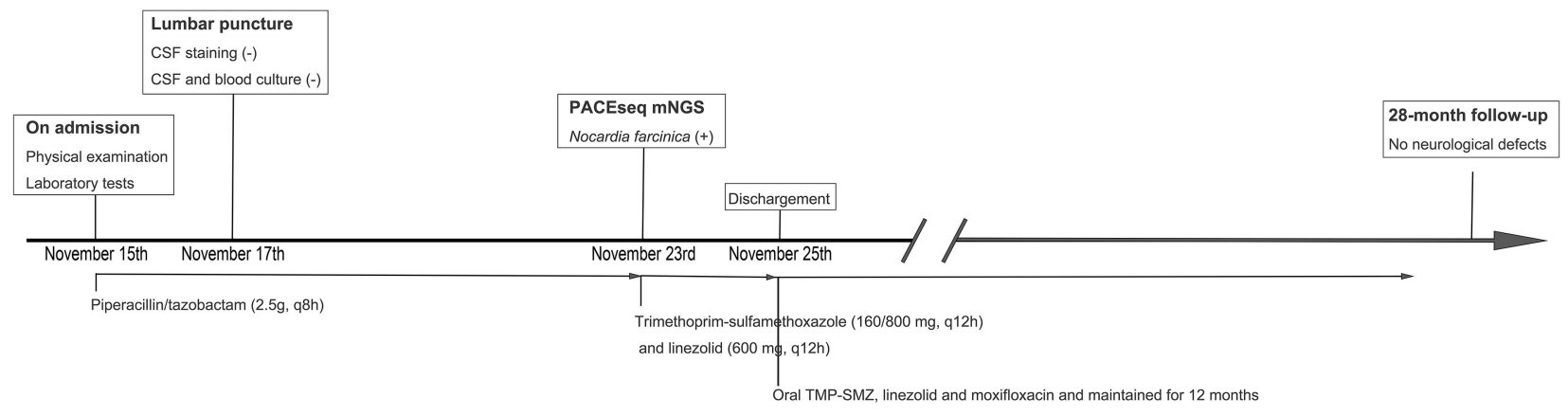

FIGURE 2 | Thoracic computed tomography (CT) scan results and timeline of the treatment. (A) Thoracic CT scan showing chronic interstitial inflammation and localized emphysema of the left upper lung. (B) Timeline of the treatment of this case.

Antibacterial treatment with piperacillin/tazobactam $(2.5 \mathrm{~g} \mathrm{q} 8 \mathrm{~h})$ was empirically started on November 15, 2018.

Lumbar puncture was then performed on November 17, 2018. The pressure was $210 \mathrm{~mm} \mathrm{H}_{2} \mathrm{O}$ (normal range 80$180 \mathrm{mmH}_{2} \mathrm{O}$ ). The appearance of the CSF is colorless and clear. CSF analysis demonstrated leukocyte at 7,000 cells $/ \mathrm{ml}$, glucose levels at $3.2 \mathrm{mmol} / \mathrm{L}$ (normal range $2.8-4.5 \mathrm{mmol} / \mathrm{L}$ ), and protein levels at $92.4 \mathrm{mg} / \mathrm{dl}$ (normal range $8-43 \mathrm{mg} / \mathrm{dl}$ ). CSF staining including Gram stain, ink stain, and acid-fast stain were all negative. Blood culture and CSF culture were both negative.

PACEseq mNGS test (Hugobiotech, Beijing, China) using CSF sample was performed to identify the pathogens on November 23, 2018. QIAamp DNA Micro Kit (QIAGEN, Hilden, Germany) was used for DNA extraction, and a library of total DNA was built with QIAseq ${ }^{\mathrm{TM}}$ Ultralow Input Library Kit for Illumina (QIAGEN, Hilden, Germany). Qubit (Thermo Fisher, MA, USA) and Agilent 2100 Bioanalyzer (Agilent Technologies, Santa Clara, USA) were used to assess the quality of the DNA library. The qualified library was finally sequenced on a Nextseq 550 platform (Illumina, San Diego, USA). After sequencing, adapters and short, low-quality, and low-complexity reads were removed from the raw data. Human DNA was also filtered out by mapping to the human reference database (hg38). The remaining reads were finally aligned to the Microbial Genome Database (ftp://ftp.ncbi.nlm.nih.gov/genomes/). N. farcinica (44 specific reads), human cytomegalovirus (2 specific reads), and human herpesvirus 4 (4 specific reads) were detected (Figure 3 ). The antibacterial regimen was changed to trimethoprimsulfamethoxazole (TMP-SMZ) (160/800 mg q12h) and linezolid (600 mg q12h). Prednisone and voriconazole were continued to be used in the treatment of pemphigus and fungal pneumonia, respectively. The symptoms of the patient improved significantly, and he was discharged on November 25.

The antibiotic regimen was changed to oral TMP-SMZ, linezolid, and moxifloxacin, and maintained for 12 months. He returned to the hospital for follow-up on March 3, 2019. MRI showed a significant reduction of brain abscess (Figure 1). 
A

Nocardia farcinica $0.0470 \%$ coverage

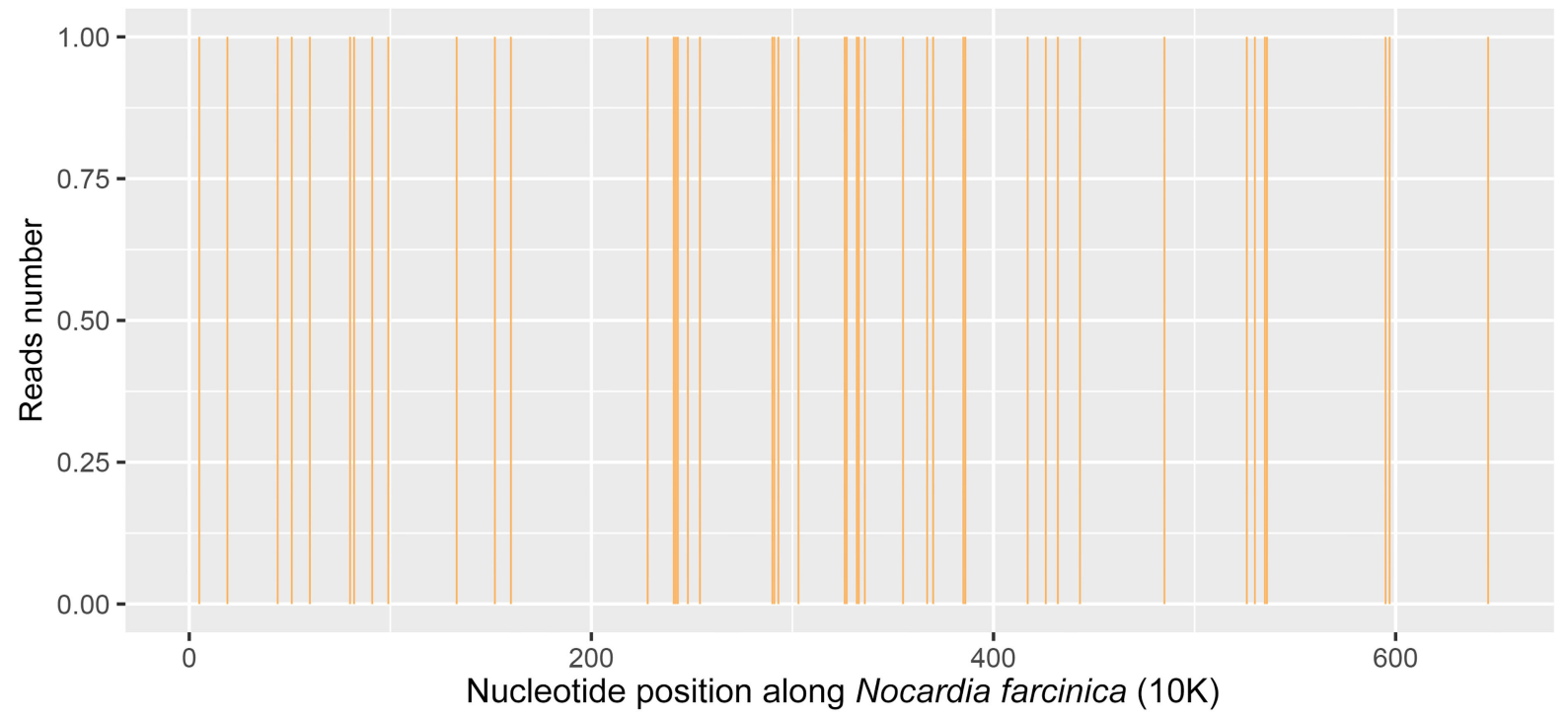

B

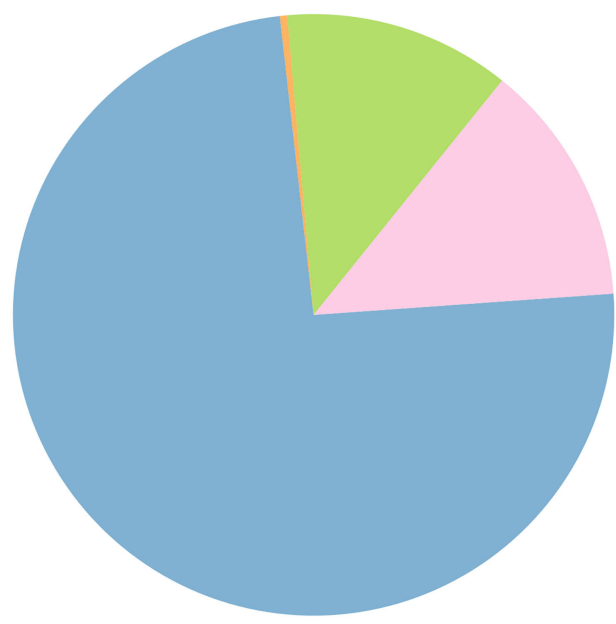

Microbe $(\mathrm{n}=11096$ reads)

Cutibacterium acnes $(n=1452,13.09 \%)$

Burkholderia ubonensis $(n=1352,12.18 \%)$

Nocardia farcinica $(n=44,0.4 \%)$

Others $(n=8248,74.33 \%)$

FIGURE 3 | Metagenomic next-generation sequencing (mNGS) results of this case. (A) Coverage of Nocardia farcinica detected by mNGS was.05\%. (B) A total of 44 specific reads of $N$. farcinica were detected by mNGS in this case.

The patient was followed up for 28 months and was currently asymptomatic without neurological defects. The timeline of the treatment is shown in Figure 2B.

\section{DISCUSSION AND CONCLUSIONS}

To the best of our knowledge, this is the first case of nocardial brain abscess with pemphigus diagnosed by mNGS without surgical interference. Huang et al. (7) reported a case of brain abscess caused by Nocardia asiatica and detected with the combination of Ziehl-Neelsen stain and mNGS. After craniotomy, mNGS identified the pathogen in the purulent fluid from the core of abscess as $N$. asiatica.
Another case reported by Zhou et al. (8) showed a idiopathic thrombocytopenic purpura patient with $N$. farcinica brain abscess diagnosed by mNGS using CSF. Unfortunately, the patient was transferred to a local hospital for treatment and lost contact.

The result of mNGS showed $N$. farcinica (44 specific reads), human cytomegalovirus (2 specific reads), and human herpesvirus 4 (4 specific reads). Cutibacterium acnes and Burkholderia ubonensis were detected as background bacteria. The role of human herpesvirus 4 (Epstein-Barr virus) in central nervous system infections is not fully resolved. EBV associated neurologic diseases include encephalitis, meningitis, cerebellitis, polyradiculomyelitis, transverse myelitis, cranial and peripheral 
neuropathies, and psychiatric abnormalities. They are usually seen in immunocompromised patients. EBV is commonly found together with other CNS infections including varicella zoster virus, cytomegalovirus, Cryptococcus, and Nocardia. Immunosuppressive state and other infections in the CNS may trigger EBV reactivation (9). Imaging findings reported on EBV encephalitis are nonspecific. Brain magnetic resonance imaging (MRI) can show normal parenchyma, leptomeningeal enhancement, and multifocal areas of hyperintensity within the subcortical white matter and deep gray nuclei (10). Cytomegalovirus infection of CNS may cause encephalitis, ventriculitis, polyradiculitis, or polyradiculomyelitis (11). Neuroimaging abnormalities include nonspecific white matter changes, periventricular enhancement, and, occasionally, mass lesions with edema and enhancement that mimic tumors (11). In this case, the MRI of the patient showed cerebellar vermis and right cerebellar mass with peripheral contrast enhancement and surrounding edema, and the symptoms of the patient significantly improved after treatment with TMP-SMZ, linezolid, and moxifloxacin, not a combination of antiviral medicines, supporting the diagnosis of brain abscess caused by Nocardia.

Nocardia is an opportunistic microorganism that exists in nature. It can be inhaled into host. Nocardia affects the lungs more frequently than the central nervous system (12). Male sex has been reported to be a risk factor for nocardial infection (13). Brain abscess caused by $N$. farcinica usually occurs in immunocompromised individuals, such as those under long-term use of immunomodulatory therapy, infected with HIV, and those who underwent cancer chemotherapy. Chronic corticosteroid therapy, especially at higher doses and prolonged duration, is associated with increased risk of opportunistic infections such as pneumocystis pneumonia.

The diagnosis of $N$. farcinica brain abscess mainly depended on bacterial culture because of its lack of characteristic imaging and clinical features. Low positive rate of bacterial culture may lead to misdiagnosis and craniotomy. It can be misdiagnosed as ischemic or hemorrhagic stroke, glioma, primary brain tumor, or metastatic malignancy $(8,14)$.

In this case, the patient was male with a history of pemphigus and had impaired immune function after long-term use of methylprednisolone. The pathogen of CSF was diagnosed to be N. farcinica by mNGS. These results suggest that mNGS is a noninvasive and reliable method for pathogen identification. As a result, the patient received timely diagnosis and sensitive antibiotic treatment with good prognosis.

Case reports and small sample cohorts described the clinical features, treatment, and prognosis of nocardial brain abscess $(5,14)$. Nocardial brain abscess occurs in people aged 50-60 years (15). Our patient is 58 years old. Brain abscess caused by $N$. farcinica tends to have multiple lesions $(16,17)$. In this case, the right cerebellum and cerebellum vermis were involved simultaneously.

\section{REFERENCES}

1. Torres OH, Domingo P, Pericas R, Boiron P, Montiel JA, Vázquez G. Infection caused by Nocardia farcinica: case report and review. Eur J Clin Microbiol
Although limited cases have been reported, in most cases, surgical excision and aspiration are considered necessary for diagnosis and treatment. Surgical aspiration is recommended in lesions larger than $2.5 \mathrm{~cm}$ in diameter or if the abscess does not decrease in size within 4 weeks (15). TMP-SMZ is the first line of therapy, and the duration is 6-12 months, or 1 year if CNS is involved. Daily TMP-SMZ prophylaxis may prevent nocardiosis and other opportunistic sensitive infections (17), while TMPSMZ treatment may not be helpful in the differential diagnosis of nocardia brain abscess. Due to the high morbidity and mortality of nocardial brain abscesses, TMP-SMZ is usually used in combination with another highly bioavailable antibiotic with good penetration to central nervous system (CNS) for the therapy of nocardial brain abscess. Antibiotics with stronger potency, namely, imipenem, amikacin, and linezolid, are recommended for the initial therapy. Recent studies have shown that linezolid can be used in combination with TMP-SMZ for its good CNS penetration (18). In this case, the patient received a combination of TMP-SMZ and linezolid regimen during admission and it was changed to oral TMP-SMZ, linezolid, and moxifloxacin for 1 year.

In conclusion, the possibility of infection with Nocardia should be kept in mind in patients who are in immunocompromised condition and exhibiting CNS infection, especially those who lack response to conventional antibiotics. CSF mNGS detection accompanied by conventional blood and CSF culture and fast-acid staining are necessary for early and accurate diagnosis. Empirical therapy including sulfonamides in combination with other high blood-brain barrier penetration antibiotics may prevent high mortality.

\section{DATA AVAILABILITY STATEMENT}

The datasets presented in this study can be found in online repositories. The names of the repository/repositories and accession number(s) can be found below: https://ngdc.cncb.ac. cn/, PRJCA007099.

\section{ETHICS STATEMENT}

The studies involving human participants were reviewed and approved by the Ethics Committee of the Affiliated Ganzhou Hospital of Nanchang University. The patients/participants provided their written informed consent to participate in this study.

\section{AUTHOR CONTRIBUTIONS}

JY and SX participated in the collection of data and drafted the manuscript. JL collected the data for case presentation. $\mathrm{XL}$ reviewed the literature and participated in its design. All the authors read and approved the final version of the manuscript.

Infectious Dis. (2000) 19:205-12. doi: 10.1007/s100960050460

2. Corti ME, Villafañe-Fioti MF. Nocardiosis: a review. Int $J$ Infectious Dis. (2003) 7:243-50. doi: 10.1016/S1201-9712(03)90 $102-0$ 
3. Mamelak AN, Obana WG, Flaherty JF, Rosenblum ML. Nocardial brain abscess: treatment strategies and factors influencing outcome. Neurosurgery. (1994) 35:622-31. doi: 10.1227/00006123-199410000-00007

4. Cassir N, Million M, Noudel R, Drancourt M, Brouqui P. Sulfonamide resistance in a disseminated infection caused by Nocardia wallacei: a case report. J Med Case Rep. (2013) 7:103. doi: 10.1186/1752-1947-7-103

5. Corsini Campioli C, Castillo Almeida NE, O'Horo JC, Challener D, Go JR, DeSimone DC, et al. Clinical presentation, management, and outcomes of patients with brain abscess due to nocardia species. Open Forum Infectious Dis. (2021) 8:ofab067. doi: 10.1093/ofid/ofab067

6. Gu W, Deng X, Lee M, Sucu YD, Arevalo S, Stryke D, et al. Rapid pathogen detection by metagenomic next-generation sequencing of infected body fluids. Nat Med. (2021) 27:115-24. doi: 10.1038/s41591-020-1105-Z

7. Huang T, Chen Y, Zhang J, He R, Qu D, Ye Q, et al. Rapid and accurate diagnosis of brain abscess caused by Nocardia asiatica with a combination of Ziehl-Neelsen staining and metagenomics next-generation sequencing. Eur J Neurol. (2021) 28:355-7. doi: 10.1111/ene.14533

8. Zhou C, Wang K, Li H, Zhang X. Idiopathic thrombocytopenic purpura with brain abscess caused by Nocardia farcinica diagnosed using metagenomics next-generation sequencing of the cerebrospinal fluid: a case report. $B M C$ Infectious Dis. (2021) 21:380. doi: 10.1186/s12879-021-06071-1

9. Martelius T, Lappalainen M, Palomäki M, Anttila VJ. Clinical characteristics of patients with Epstein Barr virus in cerebrospinal fluid. BMC Infectious Dis. (2011) 11:281. doi: 10.1186/1471-2334-11-281

10. Tselis AC. Epstein-Barr virus infections of the nervous system. Handbook Clin Neurol. (2014) 123:285-305. doi: 10.1016/B978-0-444-53488-0.00013-4

11. Marra CM. Other central nervous system infections: cytomegalovirus, Mycobacterium tuberculosis, and Treponema pallidum. Handbook Clin Neurol. (2018) 152:151-66. doi: 10.1016/B978-0-444-63849-6. 00012-8

12. Crozier JA, Andhavarapu S, Brumble LM, Sher T. First report of Nocardia beijingensis infection in an immunocompetent host in the United States. $J$ Clin Microbiol. (2014) 52:2730-2. doi: 10.1128/JCM.00588-14

13. Gorevic PD, Katler EI, Agus B. Pulmonary nocardiosis. Occurrence in men with systemic lupus erythematosus. Arch Internal Med. (1980) 140:361-3. doi: 10.1001/archinte.1980.00330150075019
14. Hofmeister MG, Dao A, Young AM. Worsening Stroke Symptoms in an 80-Year-Old Man. JAMA Neurol. (2016) 73:1017-8. doi: 10.1001/jamaneurol.2016.0421

15. Nicolosi A, Hauser WA, Musicco M, Kurland LT. Incidence and prognosis of brain abscess in a defined population: Olmsted County, Minnesota, 19351981. Neuroepidemiology. (1991) 10:122-31. doi: 10.1159/000110257

16. Zhu JW, Zhou H, Jia WQ, You J, Xu RX. A clinical case report of brain abscess caused by Nocardia brasiliensis in a non-immunocompromised patient and a relevant literature review. BMC Infectious Dis. (2020) 20:328. doi: 10.1186/s12879-020-05052-0

17. Yang M, Xu M, Wei W, Gao H, Zhang X, Zhao H, et al. Clinical findings of 40 patients with nocardiosis: a retrospective analysis in a tertiary hospital. Exp Therapeut Med. (2014) 8:25-30. doi: 10.3892/etm.2014.1715

18. Rupprecht TA, Pfister HW. Clinical experience with linezolid for the treatment of central nervous system infections. Eur J Neurol. (2005) 12:53642. doi: 10.1111/j.1468-1331.2005.01001.x

Conflict of Interest: HX is employed by Hugobiotech Co., Ltd.

The remaining authors declare that the research was conducted in the absence of any commercial or financial relationships that could be construed as a potential conflict of interest.

Publisher's Note: All claims expressed in this article are solely those of the authors and do not necessarily represent those of their affiliated organizations, or those of the publisher, the editors and the reviewers. Any product that may be evaluated in this article, or claim that may be made by its manufacturer, is not guaranteed or endorsed by the publisher.

Copyright (C) 2022 Yang, Xie, Li, Xia and Liu. This is an open-access article distributed under the terms of the Creative Commons Attribution License (CC BY). The use, distribution or reproduction in other forums is permitted, provided the original author(s) and the copyright owner(s) are credited and that the original publication in this journal is cited, in accordance with accepted academic practice. No use, distribution or reproduction is permitted which does not comply with these terms. 